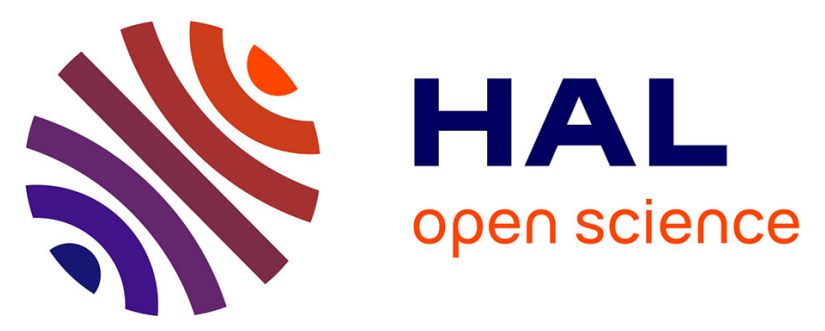

\title{
Structure-Property Relationships for Exciton and \\ Charge Reorganization Energies of Dipolar Organic Semiconductors: A Combined Valence Bond Self-Consistent Field and Time-Dependent Hartree-Fock and DFT Study of Merocyanine Dyes
}

Charlotte Brückner, Christof Walter, Matthias Stolte, Benoît Braïda, Klaus Meerholz, Frank Würthner, Bernd Engels

\section{- To cite this version:}

Charlotte Brückner, Christof Walter, Matthias Stolte, Benoît Braïda, Klaus Meerholz, et al.. Structure-Property Relationships for Exciton and Charge Reorganization Energies of Dipolar Organic Semiconductors: A Combined Valence Bond Self-Consistent Field and Time-Dependent Hartree-Fock and DFT Study of Merocyanine Dyes. Journal of Physical Chemistry C, 2015, 119 (31), pp.1760217611. 10.1021/acs.jpcc.5b06206 . hal-01627699

\author{
HAL Id: hal-01627699 \\ https://hal.science/hal-01627699
}

Submitted on 21 Nov 2017

HAL is a multi-disciplinary open access archive for the deposit and dissemination of scientific research documents, whether they are published or not. The documents may come from teaching and research institutions in France or abroad, or from public or private research centers.
L'archive ouverte pluridisciplinaire HAL, est destinée au dépôt et à la diffusion de documents scientifiques de niveau recherche, publiés ou non, émanant des établissements d'enseignement et de recherche français ou étrangers, des laboratoires publics ou privés. 


\title{
Structure-Property Relationships for Exciton and Charge Reorganization Energies of Dipolar Organic Semiconductors: A Combined Valence Bond Self-Consistent Field and Time-Dependent Hartree-Fock and DFT Study of Merocyanine Dyes
}

\author{
Charlotte Brückner, ${ }^{\dagger}$ Christof Walter, ${ }^{\dagger}$ Matthias Stolte, ${ }^{\ddagger}$ Benoît Braïda, ${ }^{\S}$ Klaus Meerholz, \\ Frank Würthner, ${ }^{\ddagger}$ and Bernd Engels*, ${ }^{*}$ \\ ${ }^{\dagger}$ Institut für Theoretische Chemie, Universität Würzburg, Emil-Fischer-Straße 42, 97074 Würzburg, Germany \\ ${ }^{\ddagger}$ Institut für Organische Chemie \& Center for Nanosystems Chemistry, Universität Würzburg, Am Hubland, 97074 Würzburg, \\ Germany \\ ${ }^{\S}$ Pierre et Marie Université, Paris 6, 4 Place Jussieu, 75005 Paris, France \\ "Department Chemie, Universität zu Köln, Luxemburgerstrasse 116, 50939 Köln, Germany
}

ABSTRACT: We present an analysis of the optoelectronic properties of merocyanine dyes by means of valence bond selfconsistent field (VBSCF), time-dependent Hartree-Fock (TD$\mathrm{HF})$, density functional theory (DFT), and high-level ab initio calculations. The electronic structure of merocyanines can be described as a superposition of two resonance structures, a neutral one and a zwitterionic one. Calculated valence bond (VB) weights for these resonance structures demonstrate the importance of strong accepting groups when increasing the weight of the zwitterionic structures of different merocyanines. The dependence of exciton and charge reorganization energies on VB weights' composition is analyzed, demonstrating that the special case of equal contributions of both structures, the socalled cyanine limit, goes along with minimal exciton and charge reorganization energies. For the latter, it is shown that the external (outer-sphere) reorganization energy plays a crucial role. Furthermore, a careful investigation of the excited-state behavior of merocyanines indicates that a possible excited-state torsion might be another important parameter for merocyaninebased optoelectronic devices, whereas internal (inner-sphere) charge reorganization energies of a variety of merocyanines are in a typical range for molecular semiconductors.

\section{INTRODUCTION}

The world's increasing energy consumption enforces the development of sustainable energy resources among which solar cells are the most promising. In contrast to their siliconbased inorganic counterparts, organic solar cells (OSCs) promise to have some major advantages such as low material and production costs and their potential fabrication on highly flexible substrates because of low processing temperatures. ${ }^{1,2}$ Although polymer-based organic bulk heterojunction $(\mathrm{BHJ})$ solar cells are easy to produce from solution, only a distribution among the polymer lengths and weights can be achieved via synthesis, making any fine-tuning of the inherent properties difficult. ${ }^{1}$ In contrast, the purification by chromatography or gradient sublimation of small organic molecules results in ultraclean products with properties determined by adjustable molecular features. ${ }^{2}$

Among many other small organic molecules, dipolar merocyanine dyes, a class of donor-acceptor (DA) chromophores with a central polymethine chain, have been successfully

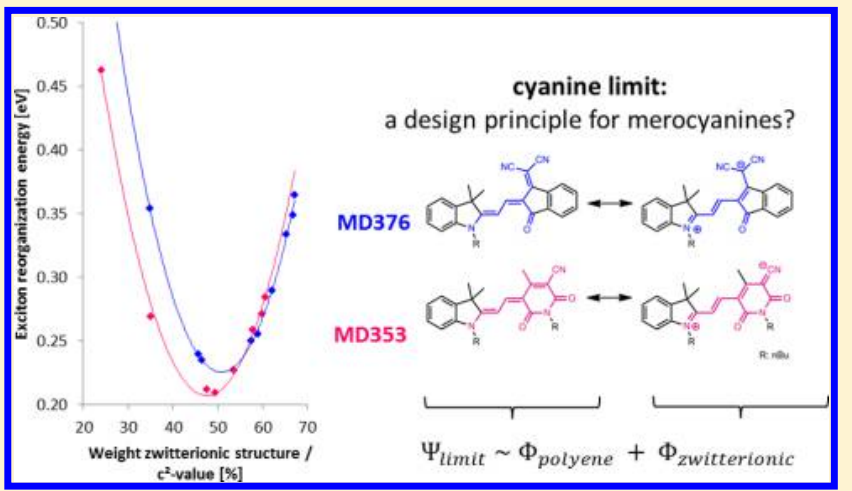

applied in recent years as p-type semiconductors in organic thin-film transistors ${ }^{3,4}$ as well as in solar cell devices. ${ }^{5-8}$ The partially high performance of these merocyanine-based devices was surprising because the use of highly dipolar molecules as organic semiconductors opposes the Bässler model for charge transport in amorphous semiconductor materials. ${ }^{9-11}$ This model states that high charge carrier mobilities are to be expected exclusively for apolar molecules, whereas a broadening of the density of states is to be expected for polar molecules because of dipolar disorder. However, for highly dipolar dyes such as merocyanine dyes, the formation of centrosymmetric dimers with vanishing dipole moments is well-established in solution $^{12,13}$ and in the bulk state, ${ }^{14,15}$ which obviously reduces the dipolar disorder in accordance with the Bässler model.

Because of the diversity of possible building blocks as well as substitution patterns, merocyanine dyes are a class of molecules 
of great structural variety with easily adjustable optical and electronic properties. ${ }^{14-25}$ To select those merocyanines that are suitable for optoelectronic and nonlinear optical applications, ${ }^{14-25}$ the so-called cyanine limit $^{16-22}$ (also called the polymethine limit) has been used as a criterion. The cyanine limit is connected with the electronic character of the ground and the first excited state of a merocyanine dye, which are commonly represented by a superposition of the neutral and zwitterionic resonance structures given in Figure la. Within the

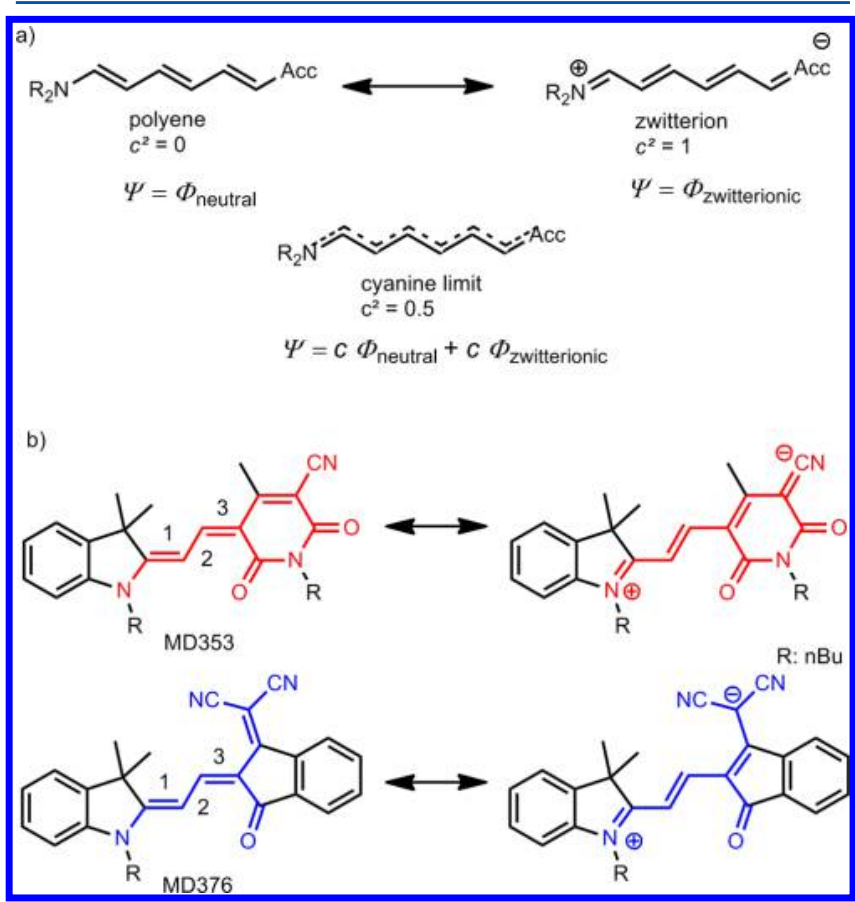

Figure 1. (a) Charge distribution in push-pull merocyanine dyes according to a simple two-state model governed by the strength of the electron-donor $\left(-\mathrm{NR}_{2}\right)$ and -acceptor (Acc) groups. The $c^{2}$ parameter allows the easy classification of a molecule between more neutral $\left(c^{2}<\right.$ $0.5)$ and more zwitterionic $\left(c^{2}>0.5\right)$ character in the electronic ground state with the special case, the cyanine limit, where both contribute equally. (b) Merocyanine dyes MD376 ${ }^{6}$ and MD353 ${ }^{8}$ investigated in this manuscript in their two resonance structures. The reduced substructures for the calculation of the reorganization energies are highlighted in color for MD376 (blue) and MD353 (red).

two-state model, the wave function of the ground state can be generally written as $\Psi=c_{1} \phi_{\text {neutral }}+c_{2} \phi_{\text {zwitterionic }}$ If the overlap between $\phi_{\text {neutral }}$ and $\phi_{\text {zwitterionic }}$ is neglected, then the wave functions of the ground and excited state can be approximated as $\Psi=\left(\left(1-c^{2}\right)\right)^{1 / 2} \phi_{\text {neutral }} \pm c \phi_{\text {zwitterionic }}$. For fully neutral structures, $c^{2}$ becomes 0 , whereas for fully zwitterionic states, it is equal to 1 . At the cyanine limit (i.e., $c^{2}=0.5$ ), both resonance structures contribute equally to both the ground and the excited state (Figure 1a).

The contributions of the two resonance structures can be determined via theoretical (e.g., by VB methods) or experimental (via electro-optical absorption (EOA) and UV/ vis spectroscopy) methods, the latter of which determine the dipole difference $(\Delta \mu)$ as well as the transition dipole moment $\left(\mu_{\mathrm{ag}}\right)$ in dilute solution between the ground and excited state. From these quantities, the $c^{2}$ value can be calculated as ${ }^{3-8,14-23}$

$$
c^{2}=\frac{1}{2}\left[1-\Delta \mu\left(4\left(\mu_{\mathrm{ag}}\right)^{2}+\Delta \mu^{2}\right)^{-1 / 2}\right]
$$

At the cyanine limit, the ground- and excited-state characters nearly coincide, leading to a variety of exceptional properties, such as strong absorption bands and the narrowest bandwidth possible. ${ }^{16-23}$ These properties result because the equilibrium geometries of both states are nearly identical, and the minimal bond length alternation ${ }^{24-26}$ in the merocyanines' central polymethine chain is found. The resulting negligible geometric change upon optical excitation when going from the ground to the excited state for a molecule at the cyanine limit was the basis for the design of our merocyanine dyes for applications in organic electronics and photovoltaics ${ }^{3-8}$ because these should lead to minimal exciton reorganization energies and the highest tinctorial strength. The strong influence of the reorganization energy on the efficiency of exciton diffusion and charge transfer is indicated, for example, by the Marcus rate equation into which the reorganization energies enter exponentially. ${ }^{27}$ Hence, small reorganization energies, as expected for merocyanine dyes at the cyanine limit $\left(c^{2}=0.5\right)$, should lead to higher device efficiencies, whereas large reorganization energies are expected for more polyene-like $\left(c^{2}<0.5\right)$ or more zwitterionic $\left(c^{2}>0.5\right)$ merocyanine dyes. Notably, such molecules are also less suitable because they possess smaller transition dipole moments.

As shown by the success of the merocyanine-based solar cells, the strategy often seems to work. However, it has never been carefully investigated whether the relationship between the cyanine limit and the exciton reorganization energy really exists and whether the $c^{2}$ parameter and concomitant dipole moment change $\Delta \mu=0$ is really a device-efficiency-determining parameter because it is not yet possible to deduce $c^{2}$ by experimental means for the solid-state material and because many other parameters (e.g., relative molecular orientation, aggregation, morphology) have a profound influence on the solar cell performance as well. With respect to these questions, we combined valence bond (VB) calculations and timedependent (TD)-HF calculations. VB theory is well-suited to investigate the electronic structure of the ground state in terms of resonance structures because its determinants closely reflect the characters of Lewis structures (e.g., the two resonance structures depicted in Figure 1). TD-HF is used to compute reorganization energies in solutions that necessitate computations of electronically excited states.

For our study, we selected two scaffolds that came up as the most successful lead structures during our experimental work on solution- and vacuum-processed $\mathrm{BHJ}$ solar cells. ${ }^{6,8}$ The first one contains the dicyanovinylindanedione heterocyclic acceptor unit (MD376) for which a number of derivatives could be developed that show power conversion efficiencies (PCEs) up to $6.1 \%$ in combination with $\mathrm{C}_{60}$ fullerene acceptors. ${ }^{5-7}$ The second one contains a pyridinedione heterocyclic acceptor unit (MD353) that also provided decently performing $\mathrm{BHJ}$ solar cells (PCEs up to $3.1 \%)^{8}$ with regard to our formerly investigated best photorefractive dyes with perfect cyaninetype absorption spectra. ${ }^{15}$

In our study, we first compared calculated VB weights with experimentally deduced $c^{2}$ values of the merocyanines MD353 and MD376. Secondly, we analyzed which structural elements of the merocyanines are responsible for their respective position relative to the cyanine limit. For this, we calculated VB weights for different fragments of MD353 and MD376. In all VB calculations, the highly polar crystal environment of the merocyanines was taken into account via an effective solvent permittivity $\left(\varepsilon_{\mathrm{r}}\right)$. Thirdly, we chose two model systems 
(highlighted in color in Figure 1b) to investigate how exciton and charge reorganization energies correlate with $c^{2}$ of a given system. Finally, we calculated exciton and charge reorganization energies for a variety of merocyanines of contrasting experimental performances in order to assess additional parameters influencing the solar cell performance such as molecular packing in the solid state as well as geometric changes within the crystal.

\section{COMPUTATIONAL DETAILS}

All alkyl substituents of MD353 and MD376 (Figure 1b) were replaced by hydrogens because their influence on the $\mathrm{VB}$ weights turned out to be insignificant. (See Results and Discussion.) The merocyanines and their model systems were optimized with B3LYP-D3/cc-pVDZ ${ }^{28-35}$ and $\mathrm{COSMO}^{36}$ (solvent: DMSO) using the Turbomole program package. ${ }^{37}$ For the VBSCF calculations, the XMVB program package ${ }^{38,39}$ was used, which is interfaced to GAMESS-US. ${ }^{40}$ All GAMESScalculations were carried out using HF/6-31G. ${ }^{41,42}$ The necessity of polarization functions was checked upon, but their use changed VB weights by only about $1 \%$. For a proper calculation of the resonance structure composition, blocklocalized wave functions using hybrid atomic orbitals were used. $^{43,44}$ To obtain a converged solution in terms of blocks, we used consistent blocks for all structures. In these calculations, the environment was again taken into account via $\mathrm{VBPCM}^{45}$

For all HF and DFT calculations, the Gaussian09 program package (revision D) was used. ${ }^{46}$ Because of charge-transfer contaminations in TD-DFT calculations, all exciton reorganization energies were calculated using TD-HF/cc-pVDZ. Charge reorganization energies were calculated with B3LYP/cc-pVDZ. PCM using state-specific solvation was used as a solvent model during the calculations of either type of reorganization energy. ${ }^{47}$ Gas-phase exciton reorganization energies of the merocyanines were calculated with SCS-CC2/cc-pVDZ. ${ }^{48,49}$ For these computations, the Turbomole program package was used.

In general, the calculation of reorganization energies involves four single-point calculations. The ground-state (neutral) reorganization energy is obtained as the difference between the ground-state (neutral) energies of the optimized groundstate (neutral) and excited-state (charged) geometries, respectively (Figures 2 and 3 ). The excited-state (cationic) reorganization energy is equivalent to the energy difference between the excited-state (cationic) energies of the optimized excited-state (charged) and ground-state (neutral) geometries, respectively. The reorganization energy is the sum of its innersphere reorganization energy (i.e., its intramolecular vibrational relaxation and its outer-sphere reorganization energy), which resumes to the solvent reorganization because of the altered electronic and geometric structure of the molecule. The two parts will be referred to hereafter as internal (inner-sphere) and external (outer-sphere) reorganization energies.

If the computations are carried out for vacuum conditions, then only the internal reorganization energies are obtained, which are solely connected with relaxation effects within the molecule. If environment effects are included, either in a molecular picture or via a continuum approach, then effects which result from the relaxation of the environment (external reorganization energies) are taken into account. ${ }^{50-52}$

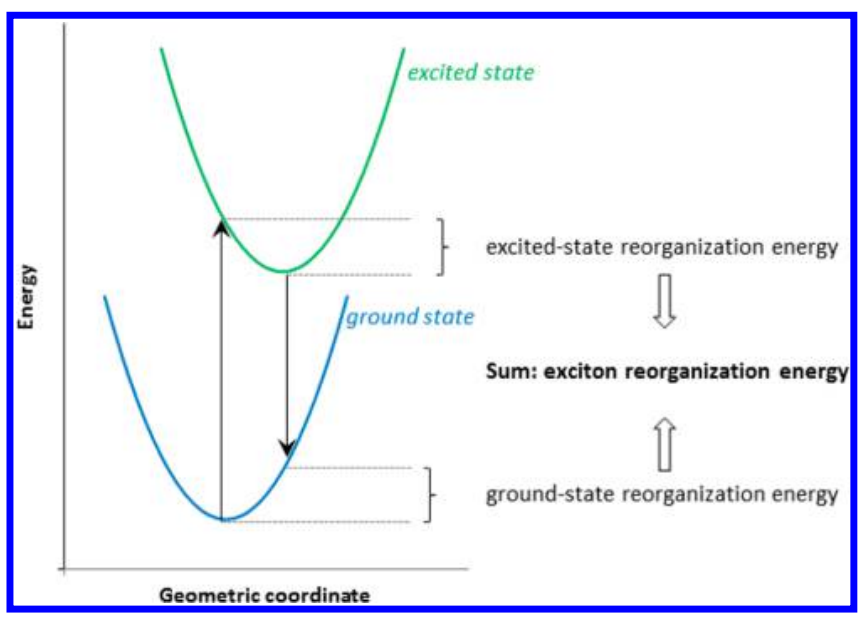

Figure 2. Exciton reorganization energy and its decomposition into the ground-state reorganization energy and excited-state reorganization energy.

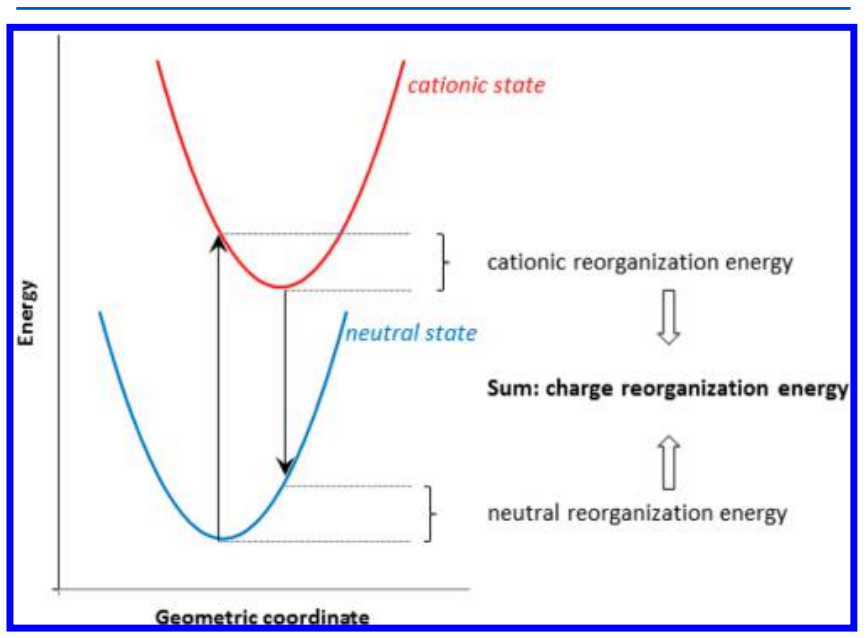

Figure 3. Charge reorganization energy and its decomposition into the neutral reorganization energy and the cationic reorganization energy.

\section{RESULTS AND DISCUSSION}

3.1. Comparisons between Computed and Measured $c^{2}$ Values. The zwitterionic VB weights of MD353 and MD376 (without alkyl substituents) are listed in Table 1 and are compared to the experimental $c^{2}$ values determined in dioxane. It was chosen because of its low permittivity and very good solubility properties. The VB weights were calculated for cyclohexane, which features a similar permittivity $(\varepsilon=2.02)$ as dioxane $(\varepsilon=2.21)$. However, in contrast to dioxane, cyclohexane does not show any effects of microsolvation, which are difficult to model by continuum solvent approaches.

The computed zwitterionic VB weights and the corresponding experimental $c^{2}$ values do agree within the error limits. The same is true for dipole moments $\mu \mathrm{g}$, and experiment and theory also coincide in the trends because both predict MD353 to be more zwitterionic than MD376. Smaller deviations probably result because the experimental values are deduced under the assumption of the two-state model and because of the neglect of the overlap between the neutral and the zwitterionic determinants. Further deviations between experiment and theory might result because the molecular properties are measured in solution but afterward corrected to the gas-phase, according to the Onsager continuum model. ${ }^{53}$ However, only 
Table 1. Comparison of Calculated VB Weights As Well As Ground State Dipole Moments $\left(\mu_{\mathrm{g}}\right)$ with the Experimentally Determined $c^{2}$ and $\mu_{\mathrm{g}}$ Values of MD353 and MD76

$\begin{array}{ccccc}\text { merocyanine } & \text { zwitterionic VB weight (\%) } & c^{2} \text { value (exp.) } & \mu_{\mathrm{g}}(\mathrm{D}) \text { (theo.) } & \mu_{\mathrm{g}} \text { (D) (exp.) } \\ \text { MD353 }^{6} & 45.6 & 0.45^{a} & 11.6^{b} & 12.8 \\ \text { MD376 }^{4 \mathrm{~b}} & 37.1 & 0.40^{a} & 7.6^{b} & 6.2\end{array}$

${ }^{a}$ Ground-state dipole moments were determined by EOA spectroscopy and corrected to the gas-phase by solvent correction within the

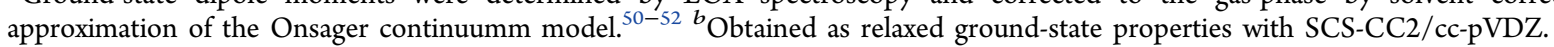

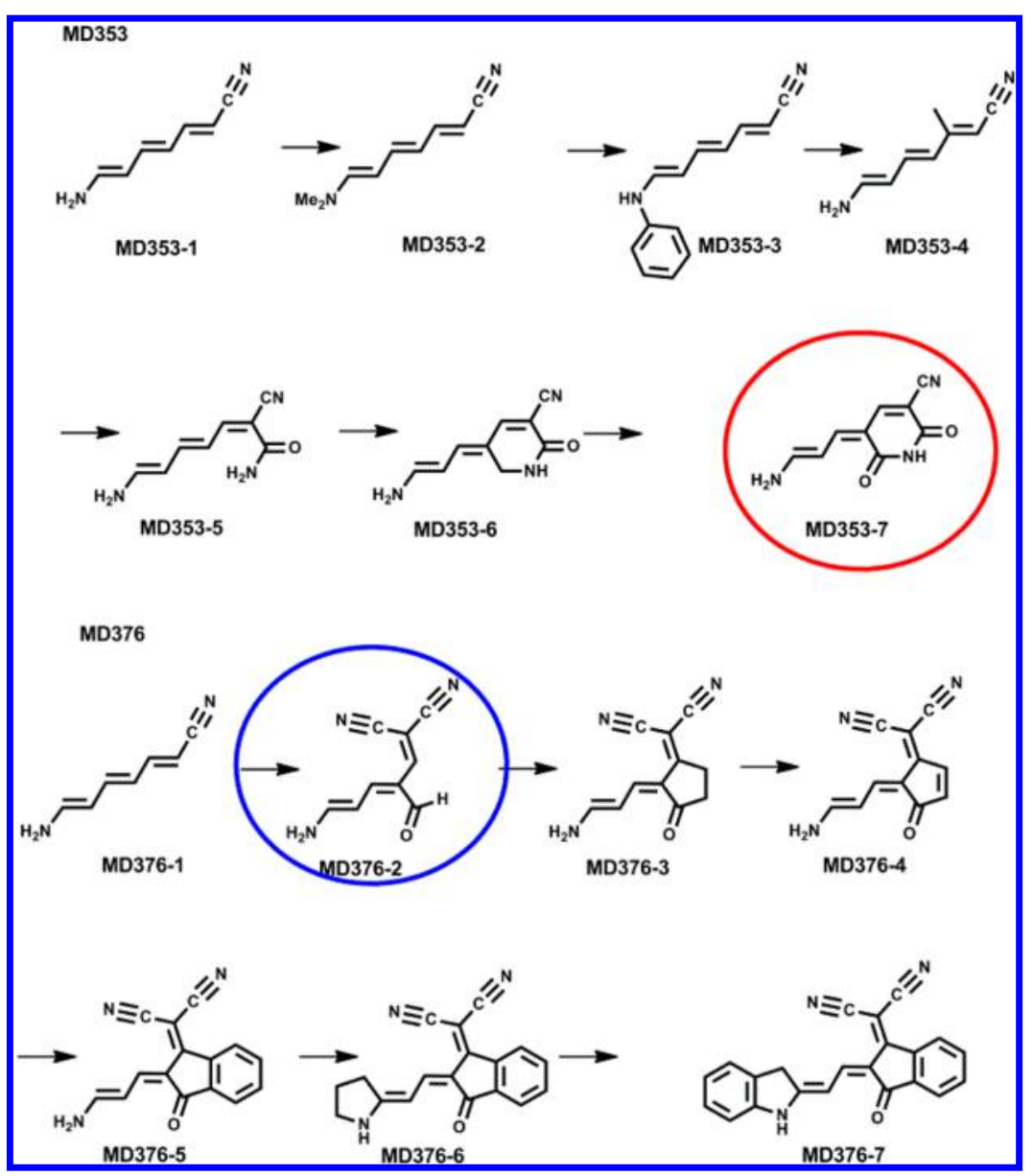

Figure 4. Stepwise buildup of MD353 (upper two rows) and MD376 (lower two rows).

the dipole moment difference $\Delta \mu$ is corrected, whereas the solution value of the transition dipole $\mu_{\mathrm{ag}}$ moment is used in the Onsager model. This justifies the use of solution values for the VB weights. Moreover, gas-phase calculations of partially charged or zwitterionic species lead to artifacts, yielding too small zwitterionic weights. Table 1 also compares the groundstate dipole moments, which coincide well.

To analyze which elements of the merocyanines are responsible for variations in the electronic structure, we varied the structures as depicted in Figure 4. Because of convergence problems in the VB computations, the indoline moiety at the donor in MD353 was not added. The corresponding VB weights computed for gas-phase $\left(\varepsilon_{\mathrm{r}}=1\right)$ and polar DMSO $\left(\varepsilon_{\mathrm{r}}\right.$ $=46.826)$ environments are given in Table 2 . The latter mimics the highly polarizable solid-state environment. Note that the values obtained for both environments show the same trends as a function of the molecular structure. Table 2 clearly shows that the decisive structural entity in MD353 is the conjugated heterocyclic acceptor group, which becomes aromatic in the zwitterionic structure (going from MD353-6 to MD353-7).
Table 2. Zwitterionic VB Weight (\%) of the Fragments of MD353 and MD376 Calculated for Gas Phase and DMSO

\begin{tabular}{llllcc} 
& \multicolumn{2}{c}{ MD353 } & & \multicolumn{2}{c}{ MD376 } \\
\cline { 2 - 3 } \cline { 5 - 6 } & $\begin{array}{c}\text { nonpolar (gas } \\
\text { phase) }\end{array}$ & $\begin{array}{c}\text { polar } \\
\text { (DMSO) }\end{array}$ & & $\begin{array}{c}\text { nonpolar (gas } \\
\text { phase) }\end{array}$ & $\begin{array}{c}\text { polar } \\
\text { (DMSO) }\end{array}$ \\
MDxxx-1 & 6.6 & 13.3 & & 6.6 & 13.3 \\
MDxxx-2 & 7.3 & 15.1 & & 40.0 & 67.5 \\
MDxxx-3 & 6.0 & 9.6 & & 38.1 & 65.3 \\
MDxxx-4 & 6.1 & 11.2 & & 27.5 & 47.3 \\
MDxxx-5 & not planar & not planar & 33.3 & 58.6 \\
MDxxx-6 & 9.3 & 26.3 & & 42.3 & 67.3 \\
MDxxx-7 & 29.7 & 62.8 & & 28.2 & 45.5 \\
\hline
\end{tabular}

The aromatization is responsible for the proximity of the final structure with respect to the cyanine limit in accordance with earlier experimental work. ${ }^{16-23}$ This becomes particularly evident upon comparison of the VB weights of the ring-closed MD353-6, which is not conjugated, in contrast to MD353-7. 


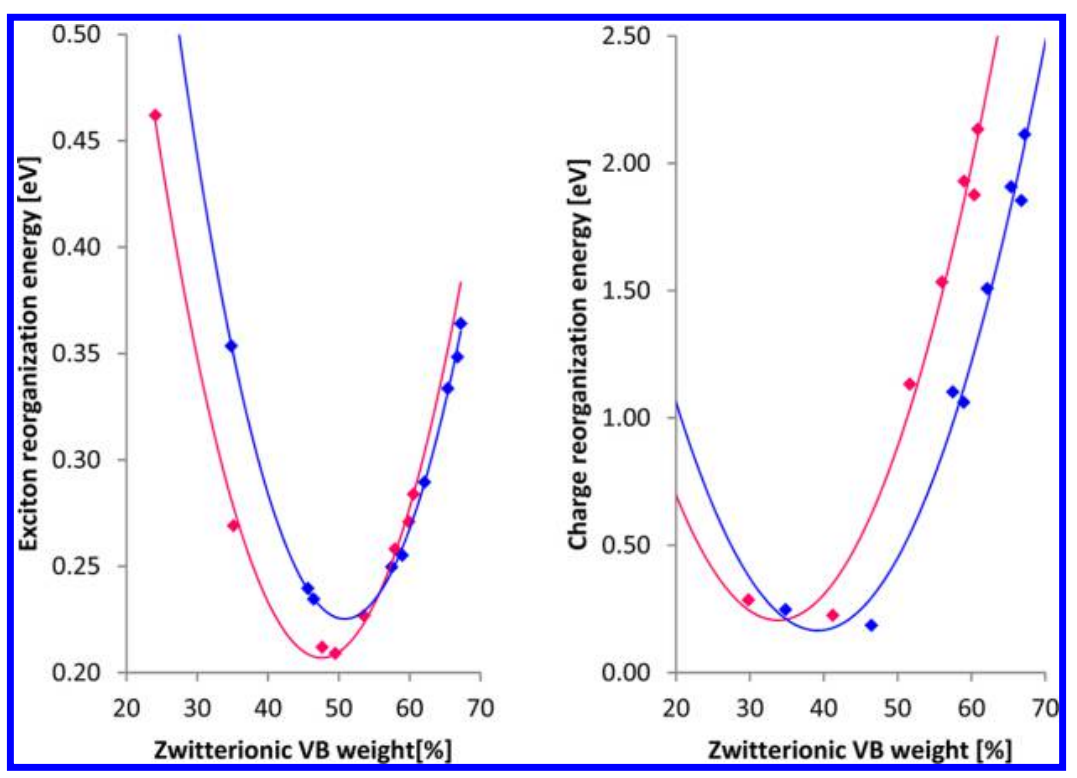

Figure 5. Correlation of (a) exciton reorganization energy and (b) total charge reorganization energy of the two model systems MD353-7 (red) and MD376-2 (blue) with their zwitterionic VB weights (TDHF/cc-pVDZ, IEFPCM with state-specific solvation). ${ }^{56}$ The lines are a guide to the eye.

The zwitterionic VB weights in Table 2 show that in the case of MD376, the combined and potentially synergetic effects of several accepting groups are needed to polarize the merocyanine toward the cyanine limit and even beyond it (structures 2, 3, 5, and 6). Additional substituent effects exerted by the ring closure at the accepting moieties show only minor changes of the VB weights' composition. In contrast, the anellation of the phenyl ring at the donor unit clearly reduces the zwitterionic character because the donating free-electron pair of the amine nitrogen ( $+\mathrm{M}$ effect) is now shared between the phenyl group and the polymethine chain. (Compare impact of phenyl group in structure 3 for MD353.)

3.2. Correlation between Electronic Structures and Exciton Reorganization Energies. To investigate the correlation between the electronic structure of a merocyanine and the reorganization energies, a series of molecules with a constantly changing electronic character (from neutral to zwitterionic) is necessary. Because variations in the substitution pattern always lead to various other effects (e.g., changes in geometry, solvation, packing, etc.) overlaying the targeted relationship between the electronic character and the reorganization energies, we carried out a series of $\mathrm{VB}$ calculations in a continuum solvation model of increasing solvent polarity. By increasing $\varepsilon_{\mathrm{r}}$, the electronic structures of the merocyanines shift from a predominantly neutral form in the ground state via the cyanine limit toward the zwitterionic form. In each solvent and thus for each VB weight composition, charge and exciton reorganization energies were calculated. For these computations, we used TD-HF, which is sufficiently accurate for trends. ${ }^{54}$ TD-DFT could not be employed because of the well-known charge-transfer-state problem. ${ }^{55}$ The calculations were carried out for structure 7 of the MD353 series (MD353-7) and structure 2 of the corresponding MD376 series (MD376-2). For the MD353 series, structure MD353-7 was chosen because all other structures do not vary sufficiently as a function of $\varepsilon_{\mathrm{r}}$ (Table 2). For the MD376 series, structures $2,3,5$, and 6 would be suitable for the investigations, but MD376-2 shows the strongest variation. The two model systems with their two resonance structures are highlighted in color in Figure $1 \mathrm{~b}$.
The computed reorganization energies include the reorganization energies of the solvent environment, which are called external reorganization energies hereafter. They are neglected in the standard two-state model that refers exclusively to molecular properties (i.e., the internal reorganization). However, external reorganization energies should be included because exciton hopping or charge transport is influenced by total reorganization energies.

The correlation of the exciton reorganization energy of the two model systems MD353-7 and MD376-2 with their zwitterionic VB weights is shown in Figure 5a. The graph clearly demonstrates the correlation of a 50:50 VB weight composition with the minimum of the exciton reorganization energy. This confirms the hypothesis that the similarity of the ground- and excited-state geometry for molecules in the cyanine limit should favor exciton diffusion. However, the dependence of the reorganization energies on the electronic character of the merocyanines is predicted to be small $(0.2 \mathrm{eV})$. Nevertheless, it should be kept in mind that the reorganization energies enter exponentially into the Marcus rate equation.

3.3. Correlation between Electronic Structures and Charge Reorganization Energies. Experimental results also seem to indicate a relationship between the cyanine limit and the charge transport efficacy. ${ }^{3-8}$ This is not predicted by the two-state model because for charge transport, the change between a neutral dye and its radical cation is the relevant process (i.e., the electronically excited state of the neutral molecule is not involved). To investigate this issue, we used the same approach as before and correlated variations of the electronic structure with the charge reorganization energies of model systems MD353-7 and MD376-2. They are shown in Figure $5 \mathrm{~b}$. Similar to the exciton reorganization energies, the charge reorganization energies also become minimal around the cyanine limit. The increase for higher zwitterionic VB weights is quite strong and can be modeled with a parabola. This quadratic fit becomes less exact in the total reorganization energies for zwitterionic VB weights smaller than $40-50 \%$. The increase of the total reorganization energies in these regions is only very weak, indicating that the minimization of the reorganization at the cyanine limit is connected only indirectly 
to the cyanine limit (see below). Nevertheless, taking both the exciton and the charge reorganization energies into account, dyes with an electronic structure around the cyanine limit are predicted to be advantageous for applications in organic photovoltaics. The total reorganization energies are smaller than the corresponding values of dyes with more polyene- or zwitterionic-like characters.

To elucidate the reasons for the existence of the minimal total charge reorganization energies, the geometries of the optimized neutral and the optimized cationic species are analyzed by calculating the bond length alternation value (BLA), ${ }^{17,20}$ which is defined as follows:

$$
\mathrm{BLA}=100\left(\frac{(\mathrm{bl} 2-0.5(\mathrm{bl} 1+\mathrm{bl} 3))}{\mathrm{bl} 2}\right)
$$

The abbreviations bl1, bl2, and bl3 designate the bond lengths of the central carbon-carbon bonds along the polymethine bridge where the enumeration starts at the donor moiety (Figure $1 \mathrm{~b}$ ). The relationship between the differences of the BLA values of the neutral and the cationic structure for both model systems and the $c^{2}$ value are shown in Figure 6. The decreasing BLA difference demonstrates that the

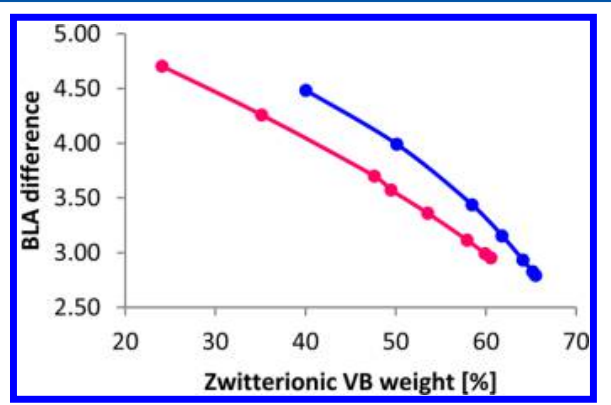

Figure 6. Dependence of the BLA of structures of MD376-2 (blue) and MD353-7 (red) upon variation of the solvent polarity.

geometrical structures of the neutral and cationic species become more and more similar with increasing zwitterionic character of the neutral species.

In contrast to exciton reorganization energies, where external reorganization energies are expected to be rather small, they should be considerably larger for charge reorganization energies (e.g., during the process of charge hopping).

Consequently, to gain more insight, the total state-specific charge reorganization energy is deconvoluted into the internal charge reorganization energy, which is connected with variations in the molecule itself, and the external charge reorganization energy, which results from solvent effects. The former can be estimated from the energy differences between the gas phase and the solvent structure both computed in the gas phase. Its dependency as a function of the electronic structure of the neutral species is given in Figure 7 together with the variation in the total reorganization energy. Subtracting these internal reorganization energies from the total charge reorganization energies (Figure $5 \mathrm{~b}$ ), we obtain the external part of the reorganization energy.

For either model system, the internal reorganization energy decreases with increasing solvent polarity. This is understandable from the decreasing geometric differences between the optimized geometries of the neutral and radical-cationic species (Figure 6) because fewer differences should lead to decreasing internal reorganization energies. In contrast, the

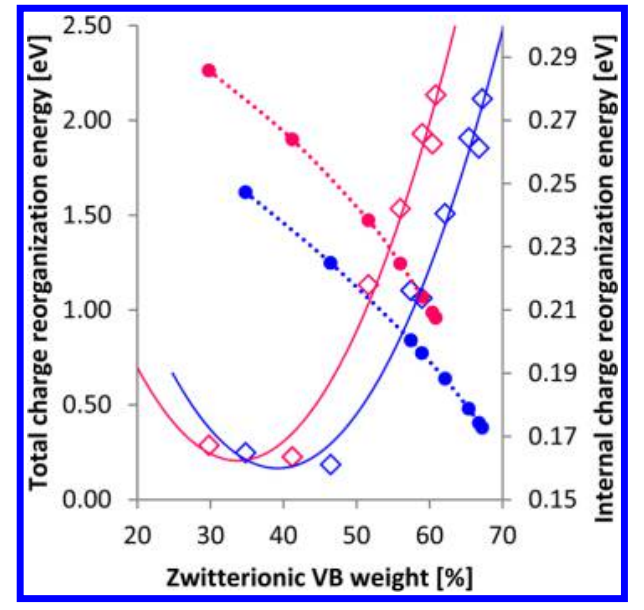

Figure 7. Total (solid lines, diamonds) and internal (dotted lines, circles) charge reorganization energies of MD376-2 (blue) and MD353-7 (red). Note the different scales for the total and the internal charge reorganization energies. All computations are carried out on the $\mathrm{B} 3 \mathrm{LYP} / \mathrm{cc}-\mathrm{pVDZ}$ level of theory.

total charge reorganization energy shows a minimum as already discussed. This is due to a compensation of the internal and the external part of the charge reorganization energy. The external reorganization energy strongly rises for increasing zwitterionic VB weights. This rise is presumably caused by the increasing reorganization effects in the environment resulting from the higher partial charges of more polar solvents. Because the electrostatic potential of the ionizable molecule changes drastically if it becomes a radical cation, after ionization the environmental charges are no longer oriented optimally, so a reorganization process is induced through the ionization of the central molecule.

Our analysis indicates that the increase in the total charge reorganization energies in solution results from a strong rise in the external reorganization energies rather than from the internal ones, which would be directly connected to the molecule. This raises the question whether our approach, which approximates the surrounding medium as a continuum, is relevant for aggregated dyes in solution, thin films, or even crystals. It results in turn to the question of whether it is possible to fold the highly anisotropic, polar, and polarizable environment of a merocyanine crystal into a single parameter, an effective epsilon, and to model thereby external reorganization. In such polar crystals, charge-charge interactions between the molecules, being of long-range character, are dominant. These strong charge-charge interactions are shielded because of the polarization of the (highly polarizable) environment. Both charge-charge interactions and polarization can be roughly modeled by an effective epsilon used in continuum solvation approaches.

In environments such as thin films or crystals, the molecule becoming the radical cation (solute) during charge transport is not surrounded by a highly mobile solvent molecules differing from the solute. In thin films or crystal environments of merocyanines, solute and solvent are identical, and because of the formation of an interaction network, they are considerably less mobile. With increasing polarity of the molecules, the interactions in an aggregate get stronger, leading to more ordered and stiffer networks. Going from fluids to amorphous thin films, the flexibility of the environment is drastically reduced. Nevertheless, because of the internal free volume, 
some reorganization of the surrounding molecules is still possible if the center molecule becomes a cation. For crystals, such reorganization motions nearly diminish. In such cases, the neighboring molecules cannot reorient because of steric restrictions in the crystal so that per definition external reorganization motions remain small. However, the chargetransfer process in these rigid environments is also hindered by a strong energetic penalty. This penalty results because initially the molecules are oriented to maximize the stabilizing interaction between the partial charges of the neutral compounds. If the central molecule is ionized within the charge transfer process, then the interaction network is massively perturbed because its partial charges have been changed completely. Because the partial charges and with this the corresponding interactions increase with increasing polarity, the resulting penalty is also expected to increase. This indicates that the predictions obtained from our model computations are also relevant for real thin-film or crystal environments.

To generalize our findings and assess any additional impact factors on the charge and exciton reorganization energies, reorganization energies were calculated for a series of six merocyanine dyes (including all substituents) composed of DA units that were applied in $\mathrm{BHJ}$ solar cells in our earlier studies. $^{5-8}$

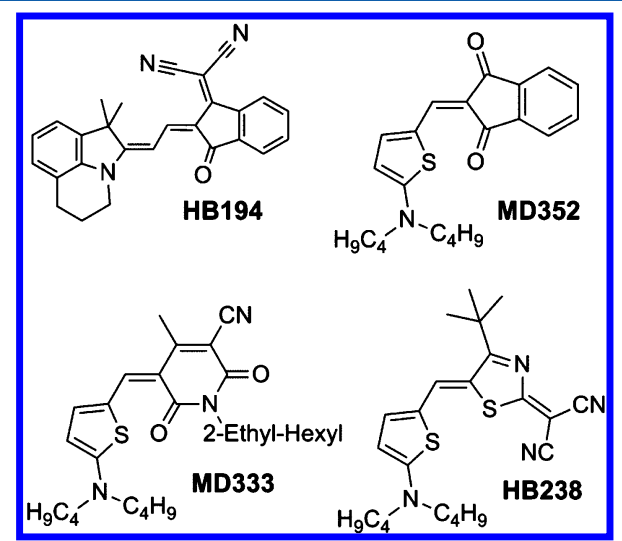

Figure 8. Structures of HB194, MD352, MD333, and HB238.

From the values for the gas-phase charge reorganization energies of different merocyanine dyes given in Table 3 , it becomes evident that they all range within the same order of magnitude, indicating that the model derived for MD353 and MD376 can be generalized. Additionally, a comparison with calculated charge reorganization energies of apolar semiconducting small organic molecules reveals that the merocyanines' charge reorganization energies are in the same order of magnitude of those of classical molecular semiconductors. Experimentally determined charge energies reorganization from UPS measurements amount to $0.097 \mathrm{eV}$ for pentacene in the gas phase and $0.109 \mathrm{eV}$ for pentacene molecules in thin films. ${ }^{57}$ Rubrene, which is more flexible, already possesses a charge reorganization energy of $0.146 \mathrm{eV} .{ }^{57}$ Copper phthalocyanine has a gas-phase charge reorganization energy of $0.063 \mathrm{eV}$, which increases to $0.106 \mathrm{eV}$ in thin films. ${ }^{58}$ The charge reorganization of zinc phthalocyanine, a dye molecule with a very small charge reorganization energy, amounts to only $0.037 \mathrm{eV}$, as determined by da Silva Filho and Brédas, et al. ${ }^{59}$

3.4. Influence of Photoinduced Torsional Motions. As opposed to the charge reorganization energies, a careful analysis of the excited-state reorganization of different merocyanines
Table 3. Gas-Phase Charge (Charge; B3LYP-D3/cc-pVDZ) and Exciton Reorganization (SCS-CC2/cc-pVDZ) Energies (ES reorg.) Calculated for a Variety of Merocyanine Dyes ${ }^{a}$

\begin{tabular}{|c|c|c|c|c|c|}
\hline merocyanine & charge $^{b}$ & ES reorg. ${ }^{c}$ & ES reorg. ${ }^{d}$ & geometry $^{e}$ & exciton $^{f}$ \\
\hline MD353 $^{6}$ & 0.17 & $h$ & 0.65 & twisted & 2.41 \\
\hline MD376 $^{6}$ & 0.12 & 0.09 & 0.13 & planar & 1.75 \\
\hline $\mathrm{HB} 194^{6}$ & 0.12 & 0.09 & 0.12 & planar & 1.75 \\
\hline MD352 $^{5}$ & 0.30 & 0.12 & 0.59 & $g$ & 2.64 \\
\hline MD333 $^{5}$ & 0.23 & 0.11 & 0.76 & $g$ & 2.51 \\
\hline${\text { HB2 } 238^{5}}^{5}$ & 0.21 & $h$ & & twisted & 2.43 \\
\hline
\end{tabular}

${ }^{a}$ For these computations, all alkyl substituents were included in the calculations. The structures are shown in Figures 1 (MD353, MD376) and $8 .{ }^{b}$ Charge reorganization energies (eV). ${ }^{c}$ Excited-state reorganization energies $(\mathrm{eV})$ : molecule remains planar. ${ }^{d}$ Excited-state reorganization energies $(\mathrm{eV})$ : twisted structure. ${ }^{e}$ Final excited-state geometry when starting from crystal structure geometry. ${ }^{f}$ Total exciton reorganization energies $(\mathrm{eV})$ for twisted excited states, including ground-state deformations and averaging over energetically almost similar enantiomers. ${ }^{g}$ No crystal structure available. ${ }^{h}$ Molecule twist instantaneously.

shows that the reorganization energies of the exciton diffusion are influenced by another effect. After excitation, some merocyanines spontaneously undergo a torsional movement when starting from the crystal structure geometry. For others, the relaxation is not spontaneous, but for all investigated merocyanines, the twisted structure is lower in energy (Table 3). Such torsional motion of photoexcited merocyanine dyes has also been not only verified by various experiments and identified as a major fluorescence-quenching pathway, but also utilized for photoreactions by light-induced electrocyclization into spiro compounds. ${ }^{60-66}$ The twisting motion predicted by our calculations is exemplified by a superposition of the planar ground-state structure and the twisted excited-state structure of MD353 in Figure 9. The photochemistry of merocyanines

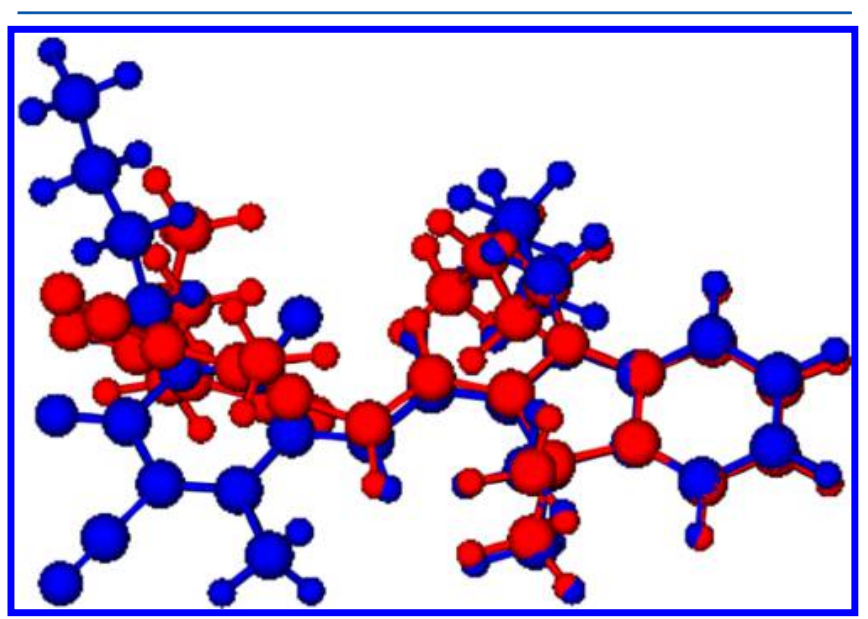

Figure 9. Planar ground-state structure (blue) and twisted excitedstate structure (red) of MD353.

along with the photoinduced motion was analyzed by $\mathrm{Xu}$ et al. ${ }^{67}$ via CASSCF and VB calculations on a push-pull model system, revealing the importance of a conical intersection. Direct experimental evidence for this interpretation of a solvent-tunable conical intersection was delivered by Kahan et al. $^{68}$

The exciton reorganization energies resulting from this induced twist motion are indeed higher by a factor of 10 than 
those from the effects discussed so far. With respect to the exciton hopping rate calculated with Marcus theory, this large reorganization energy would lead to a decrease of the rate by a factor of more than 20000 . However, the question arises whether this motion is possible in thin-film or crystal environments. Further MM calculations using AMOEBA ${ }^{69}$ showed that such an excited-state torsion would definitely not be possible in the crystal because of steric strain, even when assuming a vacancy defect in the vicinity of the twisting merocyanine. However, partial twisting motions could still be possible. Taking into account the large decrease of the hopping rate by more than four orders of magnitude in the case of a full torsion, such partial twisting motions would already be sufficient to decrease the rate of exciton hopping significantly. Consequently, the excited-state twisting motion constitutes a potential loss channel for merocyanine-dye-based organic solar cells. It is conceivable that the presence or absence of the possibility of an excited-state torsion strongly modulates effects connected with the cyanine limit. However, in the absence of an excited-state torsion, the total exciton reorganization energies ranging around $0.2 \mathrm{eV}$ are similar to or even smaller than exciton reorganization energies of other organic dyes. Calculations of Jacquemin et al. yielded around $0.4 \mathrm{eV}$ for the exciton reorganization energies of 1,8-naphthalimide fluorophores $^{70}$ and $0.71 \mathrm{eV}$ for the total exciton reorganization energy in the enole form of coumarin (calculated with TD-PBE0PCM). ${ }^{71}$

\section{CONCLUSIONS}

A comparison of calculated zwitterionic $\mathrm{VB}$ weights and the $c^{2}$ values experimentally determined from electro-optical absorption (EOA) and UV/vis spectroscopy showed good agreement for the merocyanines MD353 and MD376. This indicates that the assumptions made in the determination of the experimental $c^{2}$ values are valid. Important structural entities determining this composition are a combination of strong accepting groups and moieties potentially aromatizing in one resonance structure. Because the external exciton reorganization is small, this is valid for both the internal and the total reorganization energy.

Our investigations also indicate a relationship between the VB weights' composition and charge reorganization energy, but the computations do not show a distinct minimum at the cyanine limit. Our analysis indicates that the increase of the charge reorganization energy for structures with a predominantly zwitterionic character results from variations of the external reorganization energy, whereas the internal reorganization energy decreases as a function of the molecular zwitterionic character. Rather, they result from the response of the environment to the ionization of this molecule.

In general, our theoretical investigations support the design principle that merocyanines close to the cyanine limit should be well-suited for applications in organic solar cells. Thus, beyond the more trivial argument that the largest transition dipole moment is given at the cyanine limit and concomitantly also the highest tinctorial strength, the exciton reorganization energy is also smallest and even the charge reorganization energy can be pretty small and indeed comparable to those of the most ubiquitous unpolar organic semiconductors such as rubrene or pentacene.

However, this results from a superposition of several effects. For less zwitterionic molecules, exciton and charge reorganization energies are higher, whereas for considerably more zwitterionic ones, the rise in the external charge reorganization energies dominates. Finally, our calculations indicate that the possibility of an excited-state torsional movement of the merocyanines might be another important parameter influencing the solar cell device efficiency. Hence, it should be taken into account when designing new merocyanines for organic photovoltaics. The resulting strong influence of the environment, especially differences in the internal free volume determining the possibility of photoinduced internal torsional motions of the merocyanines, may explain why the device efficiencies of two merocyanines with nearly similar electronic structures in some cases differ considerably.

\section{AUTHOR INFORMATION}

\section{Corresponding Author}

*E-mail: bernd.engels@uni-wuerzburg.de. Phone: (+49) 93131-85394. Fax: (+49) 931-31-85331.

\section{Notes}

The authors declare no competing financial interest.

\section{ACKNOWLEDGMENTS}

We thank the DFG in the framework of the SPP 1355 for financial support and Prof. Benedetta Mennucci for fruitful discussions.

\section{REFERENCES}

(1) Gunes, S.; Neugebauer, H.; Sariciftci, N. S. Conjugated Polymerbased Organic Solar Cells. Chem. Rev. 2007, 107, 1324-1338.

(2) Mishra, A.; Bäuerle, P. Small Molecule Organic Semiconductors on the Move: Promise for Future Solar Energy Technology. Angew. Chem. Int. Ed. 2012, 51, 2020-2067.

(3) Huang, L.; Stolte, M.; Bürckstümmer, H.; Würthner, F. HighPerformance Organic Thin-Film Transistor Based on a Dipolar Organic Semiconductor. Adv. Mater. 2012, 24, 5750-5754.

(4) Liess, A.; Huang, L.; Arjona-Esteban, A.; Lv, A.; Gsänger, M.; Stepanenko, V.; Stolte, M.; Würthner, F. Organic Thin Film Transistors Based on Highly Dipolar Donor-Acceptor Polvmethine Dves. Adv. Funct. Mater. 2015, 25, 44-57.

(5) Bürckstümmer, H.; Tulyakova, E. V.; Deppisch, M.; Lenze, M. R.; Kronenberg, N. M.; Gsänger, M.; Stolte, M.; Meerholz, K.; Würthner, F. Efficient Solution-Processed Bulk Heterojunction Solar Cells by Antiparallel Supramolecular Arrangement of Dipolar Donor-Acceptor Dyes. Angew. Chem., Int. Ed. 2011, 50, 11628-11632.

(6) Kronenberg, N. M.; Steinmann, V.; Bürckstümmer, H.; Hwang, J.; Hertel, D.; Würthner, F.; Meerholz, K. Direct Comparison of Highly Efficient Solution- and Vacuum-Processed Organic Solar Cells Based on Merocyanine Dyes. Adv. Mater. 2010, 22, 4193-4197.

(7) Bürckstümmer, H.; Kronenberg, N. M.; Gsänger, M.; Stolte, M.; Meerholz, K.; Würthner, F. Tailored Merocyanine Dyes for SolutionProcessed BHJ Solar Cells. J. Mater. Chem. 2010, 20, 240-243.

(8) Steinmann, V.; Umbach, T. E.; Schädel, M.; Krumrain, J.; Lenze, M. R.; Bürckstümmer, H.; Würthner, F.; Meerholz, K. An Efficient Merocyanine/Zinc Phthalocyanine Tandem Solar Cell. Org. Electron. 2013, 14, 2029-2033.

(9) Dieckmann, A.; Bässler, H.; Borsenberger, P. M. An Assessment of the Role of Dipoles on the Density-of-States Function of Disordered Molecular Solids. I. Chem. Phvs. 1993, 99, 8136-8141.

(10) Hertel, D.; Bässler, H. Photoconduction in Amorphous Organic Solids. ChemPhysChem 2008, 9, 666-688.

(11) Schenning, A. P. H. J.; v. Herrikhuyzen, J.; Jonkheijm, P.; Chen, Z.; Würthner, F.; Meijer, E. W. Photoinduced Electron Transfer in Hydrogen-Bonded Oligo(p-phenylene vinylene)-Perylene Bisimide Chiral Assemblies. I. Am. Chem. Soc. 2002, 124, 10252-10253.

(12) Würthner, F.; Yao, S. Dipolar Dye Aggregates: a Problem for Nonlinear Optics, But a Chance for Supramolecular Chemistry. Angew. Chem. Int. Ed. 2000, 39, 1978-1981. 
(13) Würthner, F.; Yao, S.; Debaerdemaeker, T.; Wortmann, R. Dimerization of Merocyanine Dyes: Structural and Energetic Characterization of Dipolar Dye Aggregates and Implications for Nonlinear Optical Materials. I. Am. Chem. Soc. 2002, 124, 9431-9447.

(14) Würthner, F.; Meerholz, K. Systems Chemistry Approach in Organic Photovoltaic. Chem. - Eur. J. 2010, 16, 9366-9373.

(15) Würthner, F.; Wortmann, R; Meerholz, K. Chromophore Design for Photorefractive Organic Materials. ChemPhysChem 2002, 3, $17-31$.

(16) Dähne, S. Color and Constitution: One Hundred Years of Research. Science 1978, 199, 1163-1167.

(17) Marder, S. R.; Perry, J. W. Nonlinear Optical Polymers: Discovery to Market in 10 Years? Science 1994, 263, 1706-1707.

(18) Marder, S. R.; Gorman, C. B.; Meyers, F.; Perry, J. W.; Bourhill, G.; Brédas, J.-L.; Pierce, B. M. A Unified Description of Linear and Nonlinear Polarization in Organic Polymethine Dyes. Science 1994, $265,632-635$

(19) Albert, I. D. L.; Marks, T. J.; Ratner, M. A. Rational Design of Molecules with Large Hyperpolarizabilities. Electric Field, Solvent Polarity, and Bond Length Alternation Effects on Merocyanine Dye Linear and Nonlinear Optical Properties. I.Phys. Chem. 1996, 100, 9714-9725.

(20) Meyers, F.; Marder, S. R.; Pierce, B. M.; Brédas, J.-L. Electric Field Modulated Nonlinear Optical Properties of. Donor- Acceptor Polyenes: Sum-Over-States Investigation of the Relationship between Molecular Polarizabilities $(\alpha, \beta$ and $\gamma)$ and Bond Length Alternation. $\underline{I}$. Am. Chem. Soc. 1994, 116, 10703-10714.

(21) Würthner, F.; Wortmann, R.; Matschiner, R.; Lukaszuk, K.; Meerholz, K.; De Nardin, Y.; Bittner, R.; Bräuchle, C.; Sens, R. Merocyanine Dyes in the Cyanine Limit: a New Class of Chromophores for Photorefractive Materials. Angew. Chem. Int. Ed. Engl. 1997, 36, 2765-2768.

(22) Würthner, F.; Thalacker, C.; Matschiner, R.; Lukaszuk, K.; Wortmann, R. Optimization of Neutrocyanine Chromophores Based on Five-Membered Heterocycles for Photorefractive Applications. Chem. Commun. 1998, 16, 1739-1740.

(23) Wolff, J. J.; Wortmann, R. Organic Materials for Second-Order Non-Linear Optics. Adv. Phvs. Org. Chem. 1999, 32, 121-217.

(24) Marder, S. R.; Kippelen, B.; Jen, A. K. Y.; Peyghambarian, N. Design and Synthesis of Chromophores and Polymers for Electrooptic and Photorefractive Applications. Nature 1997, 388, 845-851.

(25) Mongin, O.; Porrès, L.; Moreaux, L.; Mertz, J.; Blanchard-Desce, M. Synthesis and Photophysical Properties of New Conjugated Fluorophores Designed for Two-Photon-Excited Fluorescence. Org. Lett. 2002, 4, 719-722.

(26) Beverina, L.; Pagani, G. A. $\pi$-Conjugated Zwitterions as Paradigm of Donor-Acceptor Building Blocks in Organic-Based Materials. Acc. Chem. Res. 2014, 47, 319-327.

(27) Stehr, Vera; Fink, R. F.; Engels, B.; Pflaum, J.; Deibel, C. Singlet Exciton Diffusion in Organic Crystals Based on Marcus Transfer Rates. I. Chem. Theorv Comput. 2014, 10, 1242-1255.

(28) Dirac, P. A. M. Quantum Mechanics of Many-Electron Systems. Proc. R. Soc. London, Ser. A 1929, 123, 714-733.

(29) Slater, J. C. A Simplification of the Hartree-Fock Method. Phvs. Rev. 1951, 81, 385-390.

(30) Vosko, S. H.; Wilk, L.; Nusair, M. Accurate Spin-Dependent Electron Liquid Correlation Energies for Local Spin-Density Calculations - A Critical Analysis. Can. I. Phvs. 1980, 58, 1200-1211.

(31) Becke, A. D. Density-Functional Exchange-Energy Approximation with Correct Asymptotic Behavior. Phys. Rev. A: At. Mol. Opt. Phvs. 1988, 38, 3098-3100.

(32) Lee, C.; Yang, W.; Parr, R. G. Development of the Colle-Salvetti Correlation-Energy Formula into a Functional of the Electron Density. Phvs. Rev. B: Condens. Matter Mater. Phvs. 1988, 37, 785-789.

(33) Becke, A. D. A New Mixing of Hartree-Fock and Local DensityFunctional Theories. I. Chem. Phys. 1993, 98, 5648-5652.

(34) Grimme, S. Semiempirical GGA-type Density Functional Constructed with a Long-Range Dispersion Correction. I. Comput. Chem. 2006, 27, 1787-1799.
(35) Dunning, T. H., Jr. Gaussian Basis Sets for Use in Correlated Molecular Calculations. I. The Atoms Boron through Neon and Hydrogen. I. Chem. Phvs. 1989, 90, 1007-1023.

(36) Klamt, A.; Schüürmann, G. COSMO: A New Approach to Dielectric Screening in Solvents with Explicit Expressions for the Screening Energy and its Gradient. L. Chem. Soc., Perkin Trans. 2 1993, 2, 799-805.

(37) TURBOMOLE V6.0; University of Karlsruhe and Forschungszentrum Karlsruhe GmbH: Karlsruhe, Germany, 2015; available from http://www.turbomole.com.

(38) Song, L.; Chen, Z.; Ying, F.; Song, J.; Chen, X.; Su, P.; Mo, Y.; Zhang, Q.; Wu, W. XMVB 2.0; Xiamen University: Xiamen, China, 2012.

(39) Song, L.; Mo, Y.; Zhang, Q.; Wu, W. XMVB: A Program for $A b$ Initio Nonorthogonal Valence Bond Computations. I. Comput. Chem. 2005, 26, 514-521.

(40) Schmidt, M. W.; Baldridge, K. K.; Boatz, J. A.; Elbert, S. T.; Gordon, M. S.; Jensen, J. H.; Koseki, S.; Matsunaga, N.; Nguyen, K. A.; $\mathrm{Su}, \mathrm{S}$.; et al. General Atomic and Molecular Electronic Structure System. L. Comput. Chem. 1993, 14, 1347-1363.

(41) Hehre, W. J.; Ditchfield, R.; Pople, J. A. Self-Consistent Molecular Orbital Methods. XII. Further Extensions of Gaussian-Type Basis Sets for Use in Molecular Orbital Studies of Organic Molecules. I. Chem. Phys. 1972, 56, 2257-2261.

(42) Dill, J. D.; Pople, J. A. Self-Consistent Molecular Orbital Methods. XV. Extended Gaussian-Type Basis Sets for Lithium, Beryllium, and Boron. I. Chem. Phys. 1975, 62, 2921-2923.

(43) Mo, Y.; Peyerimhoff, S. D. Theoretical Analysis of Electronic Delocalization. I. Chem. Phvs. 1998, 109, 1687-1697.

(44) Mo, Y.; et al. Charge Transfer in the Electron Donor-Acceptor Complex $\mathrm{BH}_{3} \mathrm{NH}_{3}$. L. Am. Chem. Soc. 2004, 126, 3974-3982.

(45) Song, L.; Wu, W.; Zhang, Q.; Shaik, S. VBPCM: A Valence Bond Method that Incorporates a Polarizable Continuum Model. I. Phvs. Chem. A 2004, 108, 6017-6024.

(46) Frisch, M. J.; Trucks, G. W.; Schlegel, H. B.; Scuseria, G. E.; Robb, M. A.; Cheeseman, J. R.; Scalmani, G.; Barone, V.; Mennucci, B.; Petersson, G. A.; Nakatsuji, H.; Caricato, M.; Li, X.; Hratchian, H. P.; Izmaylov, A. F.; Bloino, J.; Zheng, G.; Sonnenberg, J. L.; Hada, M.; Ehara, M.; Toyota, K.; Fukuda, R.; Hasegawa, J.; Ishida, M.; Nakajima, T.; Honda, Y.; Kitao, O.; Nakai, H.; Vreven, T.; Montgomery, J. A., Jr.; Peralta, J. E.; Ogliaro, F.; Bearpark, M.; Heyd, J. J.; Brothers, E.; Kudin, K. N.; Staroverov, V. N.; Kobayashi, R.; Normand, J.; Raghavachari, K.; Rendell, A.; Burant, J. C.; Iyengar, S. S.; Tomasi, J.; Cossi, M.; Rega, N.; Millam, J. M.; Klene, M.; Knox, J. E.; Cross, J. B.; Bakken, V.; Adamo, C.; Jaramillo, J.; Gomperts, R.; Stratmann, R. E.; Yazyev, O.; Austin, A. J.; Cammi, R.; Pomelli, C.; Ochterski, J. W.; Martin, R. L.; Morokuma, K.; Zakrzewski, V. G.; Voth, G. A.; Salvador, P.; Dannenberg, J. J.; Dapprich, S.; Daniels, A. D.; Farkas, O.; Foresman, J. B.; Ortiz, J. V.; Cioslowski, J.; Fox, D. J. Gaussian 09, revision D.01; Gaussian, Inc.: Wallingford, CT, 2009.

(47) Tomasi, J.; Mennucci, B.; Cammi, R. Quantum-Mechanical Continuum Solvation Models. Chem. Rev. 2005, 105, 2999-3093.

(48) Hellweg, A.; Grün, S.; Hättig, C. Benchmarking the Performance of Spin-Component Scaled CC2 in Ground and Electronically Excited States. Phvs. Chem. Chem. Phvs. 2008, 10, 4119.

(49) Hättig, C.; Weigend, F. CC2 Excitation Energy Calculations on Large Molecules Using the Resolution of the Identity Approximation. I. Chem. Phys. 2000, 113, 5154-5161.

(50) Malagoli, M.; Coropceanu, V.; da Silva Filho, D. A.; Brédas, J. L. A Multimode Analvsis of the Gas-Phase Photoelectron Spectra in Pligoacenes. I. Chem. Phys. 2004, 120, 7490-7496.

(51) May, V.; Kühn, O. Charge and Energy Transfer Dynamics in Molecular Systems, 3rd ed.; Wiley-VCH: Weinheim, Germany, 2011.

(52) Sánchez-Carrera, R. S.; Coropceanu, V.; da Silva Filho, D. A.; Friedlein, R.; Osikowicz, W.; Murdey, R.; Suess, C.; Salaneck, W. R.; Brédas, J.-L. Vibronic Coupling in the Ground and Excited States of Oligoacene Cations. I. Phvs. Chem. B 2006, 110, 18904-18911.

(53) Onsager, L. Electric Moments of Molecules in Liquids. I. Am. Chem. Soc. 1936, 58, 1486-1493. 
(54) Zhao, H.-M.; Pfister, J.; Settels, V.; Renz, M.; Kaupp, M.; Dehm, V.; Würthner, F.; Fink, R. F.; Engels, B. Understanding Ground and Excited State Properties of Perylene Tetracarboxylic Acid Bisimide Crystals by Means of Quantum Chemical Computations. I. Am. Chem. Soc. 2009, 131, 15660-15668.

(55) Dreuw, A.; Head-Gordon, M. Failure of Time-Dependent Density Functional Theory for Long-Range Charge-Transfer Excited States: The Zincbacteriochlorin-Bacteriochlorin and Bacteriochlorophyll-Spheroidene Complexes. I. Am. Chem. Soc. 2004, 126, 40074016.

(56) Improta, R.; Barone, V.; Scalmani, G.; Frisch, M. J. A StateSpecific Polarizable Continuum Model Time Dependent Density Functional Theory Method for Excited State Calculations in Solution. I. Chem. Phys. 2006, 125, 054103.

(57) Stehr, V.; Pfister, J.; Fink, R. F.; Engels, B.; Deibel, C. FirstPrinciples Calculations of Anisotropic Charge-Carrier Mobilities in Organic Semiconductor Crystals. Phys. Rev. B: Condens. Matter Mater. Phvs. 2011, 83, 155208.

(58) Kera, S.; Yamane, H.; Ueno, N. First-Principles Measurements of Charge Mobility in Organic Semiconductors: Valence HoleVibration Coupling in Organic Ultrathin Films. Prog. Surf. Sci. 2009, 84, 135-198.

(59) da Silva Filho, D. A.; Coropceanu, V.; Gruhn, N. E.; de Oliveira Neto, P. H.; Brédas, J.-L. Intramolecular Reorganization Energy in Zinc Phthalocyanine and Its Fluorinated Derivatives: A Joint Experimental and Theoretical Study. Chem. Commun. 2013, 49, 6069-6071.

(60) Mandal, D.; Tahara, T.; Meech, S. R. Excited-State Dynamics in the Green Fluorescent Protein Chromophore. I. Phvs. Chem. B 2004, 108, 1102-1108.

(61) Karunakaran, V.; Pérez Lustres, J. L.; Zhao, L.; Ernsting, N. P.; Seitz, O. Large Dynamic Stokes Shift of DNA Intercalation Dye Thiazole Orange has Contribution from a High-Frequency Mode. $I$. Am. Chem. Soc. 2006, 128, 2954-2962.

(62) Conyard, J.; Kondo, M.; Heisler, I. A.; Jones, G.; Baldridge, A.; Tolbert, L. M.; Solntsev, K. M.; Meech, S. R. Chemically Modulating the Photophysics of the GFP Chromophore. L. Phys. Chem. B 2011, 115, 1571-1577.

(63) Ruetzel, S.; Diekmann, M.; Nuernberger, P.; Walter, C.; Engels, B.; Brixner, T. Multidimensional Spectroscopy of Photoreactivity. Proc. Natl. Acad. Sci. U. S. A. 2014, 111, 4764-4769.

(64) Wohl, C. J.; Kuciauskas, D. Excited-state Dynamics of Spiropyran-derived Merocyanine Isomers. I. Phys. Chem. B 2005, 109, 22186-22191.

(65) Futami, Y.; Chin, M. L. S.; Kudoh, S.; Takayanagi, M.; Nakata, M. Conformations of Nitro-Substituted Spiropyran and Merocyanine Studied by Low-Temperature Matrix-Isolation Infrared Spectroscopy and Density-Functional-Theory Calculation. Chem. Phvs. Lett. 2003, 370, 460-468.

(66) Baraldi, I.; Momicchioli, F.; Ponterini, G.; Tatikolov, A. S.; Vanossi, D. Photoisomerization of Simple Merocyanines: A Theoretical and Experimental Comparison with Polyenes and Symmetric Cyanines. Phvs. Chem. Chem. Phvs. 2003, 5, 979-987.

(67) Xu, X. F.; Kahan, A.; Zilberg, S.; Haas, Y. Photoreactivity of a Push-Pull Merocyanine in Static Electric Fields: A Three-State Model of Isomerization Reactions Involving Conical Intersections. I. Phvs. Chem. A 2009, 113, 9779-9791.

(68) Kahan, A.; Wand, A.; Ruhman, S.; Zilberg, S.; Haas, Y. Solvent Tuning of a Conical Intersection: Direct Experimental Verification of a Theoretical Prediction. I. Phvs. Chem. A 2011, 115, 10854-10861.

(69) Shi, Y.; Xia, Z.; Zhang, J.; Best, R.; Wu, C.; Ponder, J. W.; Ren, P. Polarizable Atomic Multipole-Based AMOEBA Force Field for Proteins. I. Chem. Theorv Comput. 2013, 9, 4046-4063.

(70) Jacquemin, D.; Perpète, E. A.; Scalmani, G.; Ciofini, I.; Peltier, C.; Adamo, C. Absorption and Emission Spectra of 1,8-Naphthalimide Fluorophores: A PCM-TD-DFT Investigation. Chem. Phvs. 2010, 372, 61-66.

(71) Jacquemin, D.; Perpète, E. A.; Scalmani, G.; Frisch, M. J.; Assfeld, X.; Ciofini, I.; Adamo, C. Time-Dependent Density
Functional Theory Investigation of the Absorption, Fluorescence, and Phosphorescence Spectra of Solvated Coumarins. $\underline{\text { I. Chem. Phvs. }}$ 2006, 125, 164324. 